\title{
EXISTENCE AND UNIQUENESS OF MILD AND STRONG SOLUTIONS OF A VOLTERRA INTERGRODIFFERENTIAL EQUATION WITH NONLOCAL CONDITIONS
}

\author{
K. BALACHANDRAN* AND S. ILAMARAN**
}

\begin{abstract}
We prove the existence and uniqueness of mild and strong solutions of a Volterra integrodifferential equation with nonlocal initial conditions using the method of semigroups and the Banach fixed point theorem.
\end{abstract}

\section{Introduction}

Using the method of semigroups and the Banach fixed point theorem, Byszewski [2] studied about the existence and uniqueness of mild, strong and classical solutions of the following nonlocal Cauchy problem:

$$
\begin{gathered}
\frac{d u(t)}{d t}+A u(t)=f(t, u(t)), \quad t \in\left(t_{0}, t_{0}+a\right] \\
u\left(t_{0}\right)+g\left(t_{1}, \ldots, t_{p}, u(\cdot)\right)=u_{0},
\end{gathered}
$$

where $-A$ is the infinitesimal generator of a $C_{0}$ semigroup $T(t), t \geq 0$ on a Banach space $X, 0 \leq t_{0} \leq t_{1}<\ldots<t_{p} \leq t_{0}+a, a>0, u_{0} \in X$ and $f:\left[t_{0}, t_{0}+a\right] \times X \rightarrow X$, $g:\left[t_{0}, t_{0}+a\right]^{p} \times X \rightarrow X$ are given functions. Balachandran and Ilamaran [1] proved the existence and uniqueness of mild and strong solutions of the problem

$$
\begin{gathered}
\frac{d u(t)}{d t}+A u(t)=f\left(t, u(\sigma(t)), \quad t \in\left(t_{0}, t_{0}+a\right]\right. \\
u\left(t_{0}\right)+g\left(t_{1}, \ldots, t_{p}, u(\cdot)\right)=u_{0} .
\end{gathered}
$$

Moreover, Corduneanu [3] and Gripenberg [4] studied the existence of solutions of Volterra integral equations of various types using semigroups approach.

In this paper we prove two theorems about the existence and uniqueness of the mild and strong solutions of a Volterra integrodifferential equation with nonlocal intitial condition.

Received April 19, 1996.

1991 Mathematics Subject Classification. 45J05, 47D03, 47H15.

Key words and phrases. Existence of solutions, Volterra integrodifferential equation, nonlocal condition, semigroup of operators. 


\section{Preliminaries}

Consider the initial value problem

$$
\begin{aligned}
\frac{d u(t)}{d t} & =A u(t)+f(t), \quad t \in\left(t_{0}, t_{0}+a\right], \\
u\left(t_{0}\right) & =u_{0},
\end{aligned}
$$

where $f:\left[t_{0}, t_{0}+a\right] \rightarrow X, A$ is the infinitesimal generator of a $C_{0}$ semigroup $T(t), t \geq$ $0, u_{0} \in X$ and $t_{0} \geq 0$.

Throughout this paper we use the notation $I:=\left[t_{0}, t_{0}+a\right]$.

Difinition 1. A function $u$ is said to be a strong solution of problem (1), (2) on I if $u$ is differentiable almost everywhere on $I$,

$$
\begin{gathered}
\frac{d u}{d t} \in L^{1}\left(\left(t_{0}, t_{0}+a\right], X\right), u\left(t_{0}\right)=u_{0} \quad \text { and } \\
\frac{d u(t)}{d t}=A u(t)+f(t) \quad \text { a.e. on } I
\end{gathered}
$$

Theorem 1 [5]. If $X$ is a reflexive Banach space, $u_{0} \in D(A)$ and $f$ is Lipschitz continuous on I then problem (1), (2) has a unique strong solution $u$ on I given by the formula

$$
u(t)=T\left(t-t_{0}\right) u_{0}+\int_{t_{0}}^{t} T(t-s) f(s) d s, \quad t \in I .
$$

Consider the following Volterra integrodifferential equation

$$
\frac{d u(t)}{d t}+A u(t)=f(t, u(t))+\int_{t_{0}}^{t} K\left(t, s, u(s), \int_{t_{0}}^{s} H(s, \tau, u(\tau)) d \tau\right) d s, \quad t \in\left(t_{0}, t_{0}+a\right]
$$

with the nonlocal condition

$$
u\left(t_{0}\right)+g\left(t_{1}, \ldots, t_{p}, u(\cdot)\right)=u_{0}
$$

where $0 \leq t_{0}<t_{1}<\ldots<t_{p} \leq t_{0}+a,-A$ is the infinitesimal generator of a $C_{0}$ semigroup $T(t), t \geq 0$, on a Banach space $X$, and $f: I \times X \rightarrow X, g\left(t_{1}, \ldots, t_{p},.\right): X \rightarrow X$, $K: \Delta \times X \times X \rightarrow X, H: \Delta \times X \rightarrow X$ where $\Delta=\left\{(t, s): t_{0} \leq s \leq t \leq t_{0}+a\right\}$. The symbol $g\left(t_{1}, \ldots, t_{p}, u(\cdot)\right)$ is used in the sense that in the place of '.' we can substitute only elements of the set $\left\{t_{1}, \ldots, t_{p}\right\}$.

Definition 2. A continuous solution $u$ of the integral equation

$$
\begin{aligned}
u(t) & =T\left(t-t_{0}\right) u_{0}-T\left(t-t_{0}\right) g\left(t_{1}, \ldots, t_{p}, u(\cdot)\right) \\
& +\int_{t_{0}}^{t} T(t-s) f(s, u(s)) d s+\int_{t_{0}}^{t} T(t-s) \int_{t_{0}}^{s} K\left(s, \tau, u(\tau), \int_{t_{0}}^{\tau} H(\tau, \mu, u(\mu)) d \mu\right) d \tau d s, t \in I,
\end{aligned}
$$


is said to be a mild solution of problem (3), (4) on $I$.

\section{Existence of a Mild Solution}

Theorem 2. Assume that

(i) $X$ is a Banach space with norm \|\| and $u_{0} \in X$.

(ii) $0 \leq t_{0}<t_{1}<\ldots<t_{p} \leq t_{0}+a$ and $B_{r}:=\{v:\|v\| \leq r\} \subset X$.

(iii) $f: I \times X \rightarrow X$ is continuous in $t$ on $I$ and there exists a constant $L>0$ such that

$$
\left\|f\left(s, v_{1}\right)-f\left(s, v_{2}\right)\right\| \leq L\left\|v_{1}-v_{2}\right\| \quad \text { for } s \in I, \quad v_{1}, v_{2} \in B_{r} .
$$

(iv) $K: \Delta \times X \times X \rightarrow X$ is continuous and there exists a constant $K_{0}>0$ such that

$$
\left\|K\left(t, s, x_{1}, y_{1}\right)-K\left(t, s, x_{2}, y_{2}\right)\right\| \leq K_{0}\left[\left\|x_{1}-x_{2}\right\|+\left\|y_{1}-y_{2}\right\|\right]
$$

(v) $g: I^{p} \times X \rightarrow X$ and there exists a constant $G_{0}>0$ such that

$$
\begin{aligned}
& \left\|g\left(t_{1}, \ldots, t_{p}, u_{1}(\cdot)\right)-g\left(t_{1}, \ldots, t_{p}, u_{2}(\cdot)\right)\right\| \\
\leq & G_{0} \sup _{t \in I}\left\|u_{1}(t)-u_{2}(t)\right\| \quad \text { for } \quad u_{1}, u_{2} \in C\left(I, B_{r}\right) .
\end{aligned}
$$

(vi) $-A$ is the infinitesimal generator of a $C_{0}$ semigroup $T(t), t \geq 0$, on $X$

(vii) $H: \Delta \times X \rightarrow X$ is continuous and there exists a constant $H_{0}>0$ such that

$$
\left\|H\left(t, s, x_{1}\right)-H\left(t, s, x_{2}\right)\right\| \leq H_{0}\left\|x_{1}-x_{2}\right\|
$$

(viii) $M=\max _{t \in[0, a]}\|T(t)\|, N=\max _{s \in I}\|f(s, 0)\|$,

$K_{1}=\max _{t_{0} \leq s \leq t \leq t_{0}+a}\|K(t, s, 0,0)\|, \quad H_{1}=\max _{t_{0} \leq s \leq t \leq t_{0}+a}\|H(t, s, 0)\|$,

$G_{1}=\max _{u \in C(I, B)}\left\|g\left(t_{1}, \ldots, t_{p}, u(\cdot)\right)\right\|$.

(ix) $M\left(\left\|u_{0}\right\|+G_{1}+r a L+a N+K_{0} r a^{2}+K_{0} H_{0} r a^{3}+K_{0} H_{1} a^{3}+K_{1} a^{2}\right) \leq r$, and $M G_{0}+M L a+M K_{0} a^{2}+M K_{0} H_{0} a^{3}<1$.

Then problem (3), (4) has a unique mild solution on $I$.

Proof. Take $E:=C\left(I, B_{r}\right)$ and define an operator $F$ on $E$ by

$$
\begin{array}{r}
(F v)(t)=T\left(t-t_{0}\right) u_{0}-T\left(t-t_{0}\right) g\left(t_{1}, \ldots, t_{p}, v(\cdot)\right)+\int_{t_{0}}^{t} T(t-s) f(s, v(s)) d s \\
+\int_{t_{0}}^{t} T(t-s) \int_{t_{0}}^{s} K\left(s, \tau, v(\tau), \int_{t_{0}}^{\tau} H(\tau, \mu, v(\mu)) d \mu\right) d \tau d s, t \in I,
\end{array}
$$

From our assumption, we have

$$
\|(F v)(t)\| \leq\left\|T\left(t-t_{0}\right) u_{0}\right\|+\left\|T\left(t-t_{0}\right) g\left(t_{1}, \ldots, t_{p}, v(\cdot)\right)\right\|
$$




$$
\begin{aligned}
& \quad+\left\|\int_{t_{0}}^{t} T(t-s) f(s, v(s)) d s\right\|+\left\|\int_{t_{0}}^{t} T(t-s) \int_{t_{0}}^{s} K\left(s, \tau, v(\tau), \int_{t_{0}}^{\tau} H(\tau, \mu, v(\mu)) d \mu\right) d \tau d s\right\| \\
& \leq M\left\|u_{0}\right\|+M G_{1}+M \int_{t_{0}}^{t}(\|f(s, v(s))-f(s, 0)\|+\|f(s, 0)\|) d s \\
& \quad+M \int_{t_{0}}^{t} \int_{t_{0}}^{s}\left[\| K\left(s, \tau, v(\tau), \int_{t_{0}}^{\tau} H(\tau, \mu, v(\mu) d \mu)-K(s, \tau, 0,0)\|+\| K(s, \tau, 0,0) \|\right] d \tau d s\right. \\
& \leq M\left\|u_{0}\right\|+M G_{1}+M \int_{t_{0}}^{t}(L\|v(s)\|+N) d s \\
& \quad+M \int_{t_{0}}^{t} \int_{t_{0}}^{s}\left[K_{0}\|v(\tau)\|+K_{0} \int_{t_{0}}^{\tau}\|H(\tau, \mu, v(\mu))\| d \mu+K_{1}\right] d \tau d s \\
& \leq M\left(\left\|u_{0}\right\|+G_{1}+r a L+a N+K_{0} r a^{2}+K_{0} H_{0} r a^{3}+K_{0} H_{1} a^{3}+K_{1} a^{2}\right) \leq r, \text { for } v \in E .
\end{aligned}
$$

Therefore, $F E \subset E$.

Now, for every $v_{1}, v_{2} \in E$ and $t \in I$, we have

$$
\begin{aligned}
& \left\|\left(F v_{1}\right)(t)-\left(F v_{2}\right)(t)\right\| \leq\left\|T\left(t-t_{0}\right)\right\|\left\|g\left(t_{1}, \ldots, t_{p}, v_{1}(\cdot)\right)-g\left(t_{1}, \ldots, t_{p}, v_{2}(\cdot)\right)\right\| \\
& \quad+\int_{t_{0}}^{t}\|T(t-s)\|\left\|f\left(s, v_{1}(s)\right)-f\left(s, v_{2}(s)\right)\right\| d s \\
& \quad+\int_{t_{0}}^{t}\|T(t-s)\| \int_{t_{0}}^{s} \|\left[K \left(s, \tau, v_{1}(\tau) \int_{t_{0}}^{\tau} H\left(\tau, \mu, v_{2}(\mu)\right) d \mu\right.\right. \\
& \quad-K\left(s, \tau, v_{2}(\tau), \int_{t_{0}}^{\tau} H\left(\tau, \mu, v_{2}(\mu)\right) d \mu\right] d \tau d s \\
& \leq M G_{0} \sup _{t \in I}\left\|v_{1}(t)-v_{2}(t)\right\|+M L \int_{t_{0}}^{t}\left\|v_{1}(s)-v_{2}(s)\right\| d s \\
& +M K_{0} a^{2} \sup _{t \in I}\left\|v_{1}(t)-v_{2}(t)\right\|+M K_{0} \int_{t_{0}}^{t} \int_{t_{0}}^{s} \int_{t_{0}}^{\tau} H_{0}\left\|v_{1}(\mu)-v_{2}(\mu)\right\| d \mu d \tau d s \\
& \leq\left(M G_{0}+M L a+M K_{0} a^{2}+M K_{0} H_{0} a^{3}\right) \sup _{t \in I}\left\|v_{1}(t)-v_{2}(t)\right\|
\end{aligned}
$$

If we take $q:=M G_{0}+M L a+M K_{0} a^{2}+M K_{0} H_{0} a^{3}$ then

$$
\sup _{t \in I}\left\|\left(F v_{1}\right)(t)-\left(F v_{2}\right)(t)\right\| \leq q \sup _{t \in I}\left\|v_{1}(t)-v_{2}(t)\right\|
$$

with $0<q<1$.

This shows that the operator $F$ is a contraction on the complete metric space $E$. By the Banach fixed point theorem the function $F$ has a unique fixed point in the space $E$ and this point is the mild solution of problem (3), (4) on $I$. 


\section{Existence of a Strong Solution}

Definition 3. A function $u$ is said to be a strong solution of problem (3), (4) on $I$ if $u$ is differentiable a.e. on $I$,

$$
\begin{aligned}
& \frac{d u}{d t} \in L^{1}\left(\left(t_{0}, t_{0}+a\right], X\right) \\
& u\left(t_{0}\right)+g\left(t_{1}, \ldots, t_{p}, u(\cdot)\right)=u_{0}
\end{aligned}
$$

and $\left.\frac{d u(t)}{d t}+A u(t)=f(t, u(t))+\int_{t_{0}}^{t} K(t, s, u(s)), \int_{t_{0}}^{s} H(s, \tau, u(\tau)) d \tau\right) d s$, a.e. on $I$,

Theorem 3. Assume that

(i) $X$ is a reflexive Banach space with norm $\|\cdot\| \delta$.

(ii) $0 \leq t_{0}<t_{1}<\ldots<t_{p} \leq t_{0}+a$ and $B_{r}:=\{v:\|v\| \leq r\} \subset X$.

(iii) $f: I \times X \rightarrow X$ is continuous in $t$ on $I$ and there exists a constant $L>0$ such that

$$
\begin{gathered}
\left\|f\left(s_{1}, v_{1}\right)-f\left(s_{2}, v_{2}\right)\right\| \leq L\left(\left\|s_{1}-s_{2}\right\|+\left\|v_{1}-v_{2}\right\|\right) \\
\text { for } s_{1}, s_{2} \in I, v_{1}, v_{2} \in B_{r}
\end{gathered}
$$

(iv) $K: \Delta \times X \times X \rightarrow X$ is continuous and there exist constant $K_{0}>0$ such that

$$
\left\|K\left(t_{1}, s, x_{1}, y_{1}\right)-K\left(t_{2}, s, x_{2}, y_{2}\right)\right\| \leq K_{0}\left(\left|t_{1}-t_{2}\right|+\left\|x_{1}-x_{2}\right\|+\left\|y_{1}-y_{2}\right\|\right)
$$

(v) $H: \Delta \times X \rightarrow X$ is continuous and there exists a constant $G_{0}>0$ such that

$$
\left\|H\left(t_{1}, s, x_{1}\right)-H\left(t_{2}, s, x_{2}\right)\right\| \leq H_{0}\left[\left|t_{1}-t_{2}\right|+\left\|x_{1}-x_{2}\right\|\right]
$$

(vi) $g: I^{p} \times X \rightarrow X$ and there exists a constant $G_{0}>0$ such that

$$
\begin{aligned}
& \left\|g\left(t_{1}, \ldots, t_{p}, u_{1}(\cdot)\right)-g\left(t_{1}, \ldots, t_{p}, u_{2}(\cdot)\right)\right\| \\
& \leq G_{0} \sup _{t \in I}\left\|u_{1}(t)-u_{2}(t)\right\| \text { for } u_{1}, u_{2} \in C\left(I, B_{r}\right), \text { and } g\left(t_{1}, \ldots, t_{p}, \cdot\right) \in D(A)
\end{aligned}
$$

(vii) $-A$ is the infinitesimal generator of a $C_{0}$ semigroup $T(t), t \geq 0$, on $X$.

(viii) $u_{0} \in D(A)$.

(ix) $M=\max _{t \in[0, a]}\|T(t)\|, \quad N=\max _{s \in I}\|f(s, 0)\|$,

$K_{1}=\max _{t_{0} \leq s \leq t \leq t_{0}+a}\|K(t, s, 0,0)\|, \quad H_{1}=\max _{t_{0} \leq s \leq t \leq t_{0}+a}\|H(t, s, 0)\|$,

$G_{1}=\operatorname{nax}_{u \in C(I, B)}\left\|g\left(t_{1}, \ldots, t_{p}, u(\cdot)\right)\right\|$.

(x) $M\left(\left\|u_{0}\right\|+G_{1}+r a L+a N+K_{0} r a^{2}+K_{0} H_{0} r a^{3}+K_{0} H_{1} a^{3}+K_{1} a^{2}\right) \leq r$, and $M G_{0}+M L a+M K_{0} a^{2}+M K_{0} H_{0} a^{3}<1$.

Then the problem (3), (4) has a strong solution on $I$. 
Proof. Since all the assumptions of Theorem 2 are satisfied then problem (3), (4) possesses a unique mild solution belonging to $C(I ; X)$ which we denote by $u$. Now, we shall show that this mild solution is a strong solution of problem (3), (4) on $I$.

Take $K_{2}=\max _{t_{0} \leq s \leq t \leq t_{0}+a}\|K(t, s, u(s), 0)\|, \quad H_{2}=\max _{t_{0} \leq s \leq t \leq t_{0}+a}\|H(t, s, u(s))\|$

$$
L_{1}=\max _{t \in I}\|f(t, u(t))\| \text {. }
$$

Then for any $t \in I$, we have

$$
\begin{aligned}
& u(t+h)-u(t)=\left[T\left(t+h-t_{0}\right)-T\left(t-t_{0}\right)\right] u_{0} \\
& -\left[T\left(t+h-t_{0}\right)-T\left(t-t_{0}\right)\right] g\left(t_{1}, \ldots, t_{p}, u(\cdot)\right) \\
& +\int_{t_{0}}^{t_{0}+h} T(t+h-s) f(s, u(s)) d s+\int_{t_{0}+h}^{t+h} T(t+h-s) f(s, u(s)) d s \\
& -\int_{t_{0}}^{t} T(t-s) f(s, u,(s)) d s+\int_{t_{0}}^{t_{0}+h} T(t+h-s) \int_{t_{0}}^{s} K\left(s, \tau, u(\tau), \int_{t_{0}}^{\tau} H(\tau, \mu, u(\mu)) d \mu\right) d \tau d s \\
& +\int_{t_{0}+h}^{t+h} T(t+h-s) \int_{t_{0}}^{s} K\left(s, \tau, u(\tau), \int_{t_{0}}^{\tau} H(\tau, \mu, u(\mu)) d \mu\right) d \tau d s \\
& -\int_{t_{0}}^{t} T(t-s) \int_{t_{0}}^{s} K\left(s, \tau, u(\tau), \int_{t_{0}}^{\tau} H(\tau, \mu, u(\mu)) d \mu\right) d \tau d s \\
& =\left[T\left(\hat{t}+h-t_{0}\right)-T\left(t-t_{0}\right)\right] u_{0}-\left[T\left(t+h-t_{0}\right)-T\left(t-t_{0}\right)\right] g\left(t_{1}, \ldots, t_{p}, u(\cdot)\right) \\
& +\int_{t_{0}}^{t_{0}+h} T(t+h-s) f(s, u(s)) d s \\
& +\int_{t_{0}}^{t} T(t-s)[f(s+h, u(s+h))-f(s, u(s))] d s \\
& +\int_{t_{0}}^{t_{0}+h} T(t+h-s) \int_{t_{0}}^{s} K\left(s, \tau, u(\tau), \int_{t_{0}}^{\tau} H(\tau, \mu, u(\mu)) d \mu\right) d \tau d s \\
& +\int_{t_{0}}^{t} T(t-s) \int_{t_{0}}^{s+h} K\left(s+h, \tau, u(\tau), \int_{t_{0}}^{\tau} H(\tau, \mu, u(\mu)) d \mu\right) d \tau d s \\
& -\int_{t_{0}}^{t} T(t-s) \int_{t_{0}}^{s} K\left(s, \tau, u(\tau), \int_{t_{0}}^{\tau} H(\tau, \mu, u(\mu)) d \mu\right) d \tau d s \\
& \left.=T\left(t-t_{0}\right)[T(h)-I] u_{0}-T\left(t-t_{0}\right)[T(h)-I] g\left(t_{1}, \ldots, t_{p}, u(\cdot)\right)\right] \\
& +\int_{t_{0}}^{t_{0}+h} T(t+h-s) f(s, u(s)) \dot{d} s \\
& +\int_{t_{0}}^{t} T(t-s)[f(s+h, u(s+h))-f(s, u(s))] d s \\
& +\int_{t_{0}}^{t_{0}+h} T(t+h-s) \int_{t_{0}}^{s}\left[K\left(s, \tau, u(\tau), \int_{t_{0}}^{\tau} H(\tau, \mu, u(\mu)) d \mu\right)-K(s, \tau, u(\tau), 0)\right] d \tau d s
\end{aligned}
$$




$$
\begin{aligned}
& +\int_{t_{0}}^{t_{0}+h} T(t+h-s) \int_{t_{0}}^{s} K(s, \tau, u(\tau), 0) d \tau d s \\
& +\int_{t_{0}}^{t} T(t-s) \int_{t_{0}}^{s}\left[K\left(s+h, \tau, u(\tau), \int_{t_{0}}^{\tau} H(\tau, \mu, u(\mu)) d \mu\right)\right. \\
& \left.\quad-K\left(s, \tau, u(\tau), \int_{t_{0}}^{\tau} H(\tau, \mu, u(\mu)) d \mu\right)\right] d \tau d s \\
& +\int_{t_{0}}^{t} T(t-s) \int_{s}^{s+h}\left[K\left(s+h, \tau, u(\tau), \int_{t_{0}}^{\tau} H(\tau, \mu, u(\mu)) d \mu\right)-K(s+h, \tau, u(\tau), 0)\right] d \tau d s \\
& +\int_{t_{0}}^{t} T(t-s) \int_{s}^{s+h} K(s+h, \tau, u(\tau), 0) d \tau d s
\end{aligned}
$$

Using our assumptions we observe that

$$
\begin{aligned}
\|u(t+h)-u(t)\| \leq & h M\left\|A u_{0}\right\|+h M\left\|A g\left(t_{1}, \ldots, t_{p}, u(\cdot)\right)\right\|+h M L_{1} \\
& \quad+M L a h+M L \int_{t_{0}}^{t}\|u(s+h)-u(s)\| d s \\
& +M K_{0} H_{2} a^{2} h+M K_{2} a h+M K_{0} a^{2} h+M K_{0} H_{2} a^{2} h+M K_{2} a h \\
& \leq P h+M L \int_{t_{0}}^{t}\|u(s+h)-u(s)\| d s,
\end{aligned}
$$

where $P:=M\left\|A u_{0}\right\|+M\left\|A g\left(t_{1}, \ldots, t_{p}, u(\cdot)\right)\right\|+M L_{1}+M L a$

$$
+M K_{0} H_{2} a^{2}+M K_{2} a+M K_{0} a^{2}+M K_{0} H_{2} a^{2}+M K_{2} a \text {. }
$$

Using Gronwall's inequality, we get

$$
\|u(t+h)-u(t)\| \leq P h e^{M L a} \quad \text { for } \quad t \in I .
$$

Therefore, $u$ is Lipschitz continuous on $I$.

The Lipschitz continuity of $u$ on $I$ combined with (iii) give that $t \rightarrow f(t, u(t))$ is Lipschitz continuous on $I$. Also, by assumption (iv) and (v) $t \rightarrow \int_{t_{0}}^{t} K(t, s, u(s)$, $\left.\int_{t_{0}}^{s} H(s, \tau, u(\tau)) d \tau\right) d s$ is Lipschitz continuous on $I$. Using Theorem 1 we observe that the equation

$$
\begin{aligned}
\frac{d v(t)}{d t}+A v(t) & =f(t, u(t))+\int_{t_{0}}^{t} K\left(t, s, u(s), \int_{t_{0}}^{s} H(s, \tau, u(\tau)) d \tau\right) d s, \quad t \in\left(t_{0}, t_{0}+a\right] \\
v\left(t_{0}\right) & =u_{0}-g\left(t_{1}, \ldots, t_{p}, u(\cdot)\right)
\end{aligned}
$$

has a unique strong solution $v$ on $I$ satisfying the equation

$$
\begin{aligned}
v(t) & =T\left(t-t_{0}\right) u_{0}-T\left(t-t_{0}\right) g\left(t_{1}, \ldots, t_{p}, u(\cdot)\right) \\
& +\int_{t_{0}}^{t} T(t-s) f(s, u(s)) d s+\int_{t_{0}}^{t} T(t-s) \int_{t_{0}}^{s} K\left(s, \tau, u(\tau), \int_{t_{0}}^{\tau} H(\tau, \mu, u(\mu) d \mu) d \tau d s\right. \\
& =u(t), \quad t \in I .
\end{aligned}
$$


Consequently, $u$ is a strong solution of problem (3), (4) on $I$.

\section{References}

[1] K. Balachandran and S. Ilamaran, "Existence and uniqueness of mild and strong solutions of a semilinear evolution equation with nonlocal condition," Indian J. Pure and Appl. Math. 25 (1994), 411-418.

[2] L. Byszewski, "Theorems about the existence and uniqueness of solutions of a semilinear evolution nonlocal Cauchy problem," J. Math. Anal. and Appl., 162. 2 (1991), 494-505.

[3] C. Corduneanu, "Integral Equations and Applications," Cambridge University Press, 1991.

[4] G. Gripenberg, S. O. Londen, O. Staffens, "Volterra Integral and Functional Equations," Cambridge University Press, 1990.

[5] A. Pazy, "Semigroups of Linear Operators and Applications to Partial differential equations," Springer-Verlag, NewYork, 1983.

Department of Mathematics * Bharathiar University, Coimbatore-641 046 ** CBM College, Coimbatore-641042 Tamil Nadu, India. 